\title{
Aspectos pesqueros y biología reproductiva de Hemiancistrus guahiborum, en un pedregal del río Bita, Puerto Carreño - Colombia
}

\author{
Fisheries aspects and reproductive biology of Hemiancistrus \\ guahiborum, in a scree in the Bita river, Puerto Carreño - \\ Colombia
}

\section{Aspectos pesqueiros e biologia reproductiva de Hemiancistrus guahiborum, num pedregal do rio Bita, Puerto Carreño - Colombia}

\author{
Rosa E. Ajiaco-Martínez", Hernando Ramírez-Gil', José A. Arias-Castellanos²
}

1 B.M. MSc; Grupo Evaluación, manejo y conservación de recursos pesqueros, Facultad de Ciencias Básicas e Ingeniería,
Universidad de los Llanos.
2 B. MSc, PhD Grupo de estudio en peces ornamentales Chamú Jialré, Instituto de Acuicultura, Universidad de los Llanos
Email:reajiaco@gmail.com

Recibido: Noviembre 18 de $2011 \quad$ Aceptado: Septiembre 27 de 2012

\begin{abstract}
Resumen
En el pedregal El Alizal del río Bita en Puerto Carreño, Vichada, se estudio la pesquería y la biología reproductiva de la especie Hemiancistrus guahiborum. Para lo cual se definió la unidad económica de pesca (UEP) y se calcularon los parámetros de esfuerzo y captura. Igualmente se estimó la densidad de peces en el pedregal y su patrón de distribución mediante el método de la cuadrícula. A partir de la determinación del sexo y estado de madurez gonadal de ejemplares capturados mensualmente, se estableció la época de reproducción y se estimaron el factor de condición total (K) y el factor de condición somático (K'), el índice gonadosomático, la fecundidad absoluta $(\mathrm{F})$ y relativa a peso y la talla media de maduración sexual ( $\mathrm{L}_{50 \%}$ ). La UEP la constituye un pescador y su careta, con capturas por unidad de esfuerzo de $28 \pm 18$ ejemplares por faena, en faenas de $52 \pm$ 20 min. La densidad media fue de 0.06 ejemplares $/ \mathrm{m}^{2}$, con una relación varianza / media de 8.86. En todos los meses muestreados se observaron ejemplares maduros y no se presentó variación significativa de los factores de condición $\mathrm{K}$ y $\mathrm{K}^{\prime}$, ni del índice gonadosomático entre meses. La F se estableció en $48 \pm 19$ ovocitos y la fecundidad relativa en5.3 \pm 0.3 ovocitos/g. La talla $L_{50 \%}$ de madurez se estimo en $6.4 \mathrm{~cm}$ de Longitud Estándar para hembras y en $7.5 \mathrm{~cm}$ para machos. Se observó cuidado parental del macho. Como estrategias de manejo se propone: mantener el período de veda actual, establecer la talla de captura en $6 \mathrm{~cm}$ de Longitud Estándar, prohibir el comercio de hembras ovadas, establecer áreas de cría dentro de los pedregales, rotar la pesca de los pedregales e incentivar los procesos de cría en cautiverio de la especie.
\end{abstract}

Palabras clave: cucha roja, densidad, factor de condición, fecundidad, talla media de madurez, unidad económica de pesca

\begin{abstract}
In the scree El Alizal of the Bita river, Puerto Carreño, Vichada, was studied fisherie and reproductive biology of the species Hemiancistrus guahiborum. The fisheries economic unit (FEU) and the parameters of effort and fishing were calculated.
\end{abstract}


Likewise, the fish density in the scree and its distribution pattern were determined using the grid method. By observing the gender and gonadal mature status of monthly caught samples, the breeding season was determined and the total condition factor $(K)$ and the somatic condition factor $\left(K^{\prime}\right)$, the gonadosomatic index, the absolute fecundity $(F)$ and relative fecundity at weight and the mean size of sexual maturity $\left(\mathrm{L}_{50 \%}\right)$ were calculated. The FEU was composed by a fisherman and its mask, who using lung freediving, captured $28 \pm 18$ individuals / fishing, in fishing tasks that lasted $52 \pm 20$ min. The mean density in the scree river was 0.06 individuals $/ \mathrm{m}^{2}$ with a relation variance /mean of 8.86 . Mature individuals were observed in all sampled months. Not significant variation in the condition factor ( $\mathrm{K}$ and $\mathrm{K}^{\prime}$ ) either gonadosomatic index between months was observed. The $\mathrm{F}$ was established in $48 \pm 19$ oocytes and the relative fecundity was $5.3 \pm 0.3$ oocytes / g. The maturity size $\mathrm{L}_{50 \%}$ was determined in $6.4 \mathrm{~cm}$ and $7.5 \mathrm{~cm}$ standard length for females and males, respectively. Parental care in nest was observed in the males. As management strategies are suggested the follows: keep the period of current fishing ban, establish the fishing size in $6 \mathrm{~cm} \mathrm{SL}$, ban the trade of mature females, establish breeding areas in the scree rotate the fishing in the scree river and stimulate the captive breeding of the species.

Keywords: condition factor,density, fecundity,fishing economic unit, mean size of maturity, red cucha

\section{Resumo}

No calhaus El Alizal do rio Bita em Puerto Carreño, Vichada, foi estudada a pescaria e biología reprodutiva da espécie Hemiancistrus guahiborum. Assim, definiou-se a unidade econômica da pesca (UEP) e foram calculados os parâmetros de esforço e captura. Também estimou-se a densidade de peixes e seu padrão de distribuição pelo método de quadrícula. Com base na determinação do sexo e estádio de maturação gonadal dos peixes capturados mensalmente, estabeleceu-se a época de reprodução e foi estimado o fator de condição total (K) e fator de condição somáticas (K '), o índice gonadossomático, a fecundidade absoluta (F) e relativa ao peso e tamanho médio de maturação sexual (L50\%). A UEP é constituida por um pescador e sua máscara de mergulho, com captura por unidade de esforço de $28 \pm 18$ exemplares em periodos de $52 \pm 20$ min. A densidade média foi de 0,06 exemplares $/ \mathrm{m}^{2}$, com uma proporção variançã /média de 8.86 . Em todos os meses de amostragem foram observados adultos maturos e não mostraram variação significativa dos factores de condições K e K', nim do índice gonadossomático entre os meses. A F foi de $48 \pm 19$ ovócitos e a fecundidade relativa em 5.3 \pm 0.3 ovócitos / g. O tamaño medio de maduração L50\% foi estimada em $6.4 \mathrm{~cm}$ de comprimento padrão para as femias e $7 \mathrm{~cm}$ para os machos. Foi observado cuidado parental dos machos. Como estratégia de manejo pesqueiro é proposto: manter o período de proibição atual, estabelecer o tamanho de captura de 6 centímetros de comprimento padrão, proibir o comercio de fêmeas ovadas, estabelecer áreas de reprodução dentro dos calhaus, rotar a pesca dos calhaus e começar processos de reprodução em cativeiro da espécie

Palavras chaves:cascudo vermelho, comprimento médio de maduração, densidade, fator de condição, fecundidade, unidade econômica de pesca

\section{Introducción}

La explotación de especies ícticas con fines ornamentales es una actividad que desde los años 1950 (Castro, 2005), ha generado ingresos y empleo a los habitantes de zonas alejadas de los grandes centros de consumo, como es Puerto Carreño, Vichada. Esta zona, con abundante oferta de recursos hídricos, entrega al mercado una amplia diversidad de especies de peces apreciados a nivel internacional, dentro de ellos los pertenecientes a la familia Loricariidae, denominados comercialmente como cuchas.

Dentro de los loricaridos de la subfamilia Hypostominae, se ubica la tribu Ancistrini, con 24 géneros (Armbruster, 2004), uno de ellos Hemiancistrus, con 15 especies validas (Souza et al., 2008), las cuales se distribuyen desde Panamá hasta el sur de Brasil (Cardoso y Silva, 2004), y se caracterizan por tener un opérculo en forma de hoz y puntos oscuros o luminosos sobre un cuerpo oscuro (Cardoso y Lucinda, 2003; Armbruster y Werneke, 2005; Armbruster, 2008).
Hemiancistrus guahiborum, la especie objeto de estudio, habita de manera endémica los pedregales del río Bita, en el área de influencia de Puerto Carreño. Los pedregales son parches de rocas precámbricas de color pardo rojizo formados por trozos de cornisas de corazas ferruginosas originadas a partir de aglomeraciones de fragmentos arenosos cementados con oxido de hierro, los cuales afloran en algunas partes y se desprenden por acción de la erosión de las lluvias y del río. Los pedregales se proyectan desde la orilla y llegan máximo a la parte central del río (Ramírez-Gil y Ajiaco-Martínez, 2008). Son porosos y así ofrecen refugio y alimento a las varias especies de peces que los habitan.

La explotación de los peces en los pedregales se realiza de forma desordenada e ilimitada, sin que pescadores ni comerciantes consideren que es un recurso finito $y$ que su extracción debe racionalizarse. La información sobre las capturas y comercialización es fragmentaria. Ramírez-Gil et al. (2001), reportaron que en el año 1999 se pescaron en la zona aproximadamente 9000 
ejemplares. Para el 2007 se comercializaron 6733 unidades, en el 200811210 ejemplares y en el 2009 8321 peces (MADR-CCI, 2008; SIPA, 2008, 2009).

No se sabía hasta ahora sobre aspectos de la pesquería, como la abundancia y distribución en los pedregales, que permitiera establecer si la población existente puede seguir sosteniendo la explotación y cuáles podrían ser las estrategias de manejo, tampoco sobre la biología de la especie, en especial de los indicadores reproductivos.

Por lo anterior era necesario dirigir esfuerzos para acopiar la información base (Kapetsky, 1982; Seijo et al., 1997; FAO, 2003), y analizada, recomendar medidas de ordenamiento que garanticen que laespecie permanezca en un nivel biológico y ambientalmente seguro y productivo y respondiendo a las expectativas sociales y económicas de los pescadores de la región(Caddy y Mahon, 1996).

\section{Materiales y métodos}

Área de estudio. Para definir el pedregal de estudio se realizaron recorridos en lancha por el río Bita en la temporada de aguas bajas en un tramo de $70 \mathrm{~km}$, desde las coordenadas $6^{\circ} 10^{\prime} 55^{\prime \prime} \mathrm{N}$ y $67^{\circ} 43^{\prime} 11^{\prime \prime} \mathrm{W}$ hasta la confluencia de este en el río Orinoco. Durante tales exploraciones con el empleo de una ecosonda (Eagle Magna II Plus), se ubicaron los pedregales y se georeferenciaron con GPS (Garmin, modelo MAP 76CSX). La información recolectada fue complementada mediante entrevistas con los grupos de pescadores que realizan faenas en ellos, estableciéndose finalmente cual de todos era el de mayor frecuencia de pesca y procediéndose así a seleccionarse el pedregal El Alizal localizado a $21.3 \mathrm{~km}$ aguas arriba de la desembocadura del río Bita en el río Orinoco $\left(6^{\circ} 12^{\prime} 0.8^{\prime \prime} \mathrm{N} ; 67^{\circ} 38^{\prime}\right.$ 20.5" O) (Figura 1). El área total de El Alizal se estableció midiendo su longitud (paralela a la corriente) y ancho con el GPS. También se preciso la longitud del mismo explotada por los pescadores, la cual fue de 45 $\mathrm{m}$ de longitud.

Determinación de parámetros pesqueros. En la temporada de aguas bajas de 2009 se realizaron dos expediciones de cinco días cada una, al inicio de la temporada (enero) y al final de la misma (abril), en las cuales se acompaño las jornadas de los pescadores que aprovechan el recurso en El Alizal, pudiéndose establecerla Unidad de Esfuerzo de Pesca (UEP), el número y duración de las inmersiones realizadas por cada pescador en cada faena y la cantidad de peces de la especie obtenidos por inmersión y faena. Con estos datos se estimaron los estadísticos descriptivos (promedio \pm desviación estándar), teniendo la faena como medida de esfuerzo (Bustamante et al., 2010), y se estimó la captura por unidad de esfuerzo, parámetro utilizado comúnmente como índice de abundancia de la población bajo presión de pesca (King, 1995; $\mathrm{FAO}, 2001$ ).

Establecimiento de la densidad y distribución de la población. En El Alizal se delinearon, con GPS, tres transeptos desde la orilla hasta el centro del río, ubicando el primero en el límite del pedregal aguas arriba $\left(T_{1}\right)$, el siguiente en la parte media $\left(T_{2}\right)$ y el tercero al finalizar el pedregal aguas abajo $\left(\mathrm{T}_{3}\right)$, los transeptos

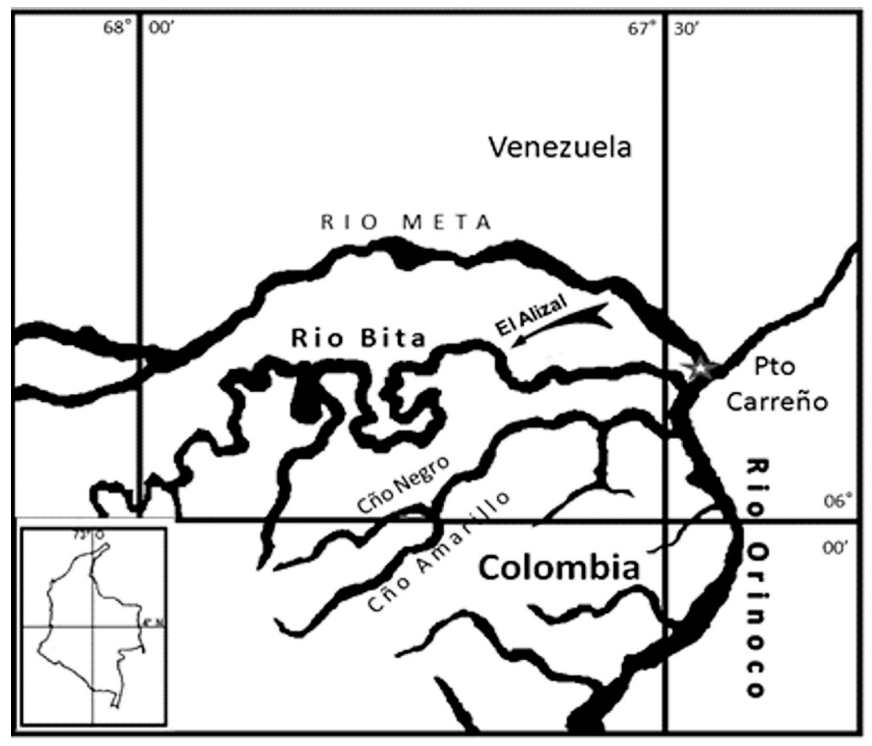

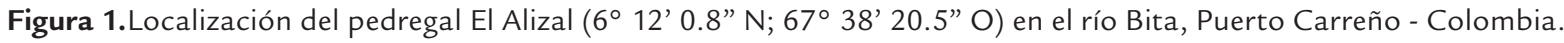


fueron relacionados con un transepto perpendicular a los mismos en el sentido de la corriente $\left(T_{4}\right)$, ubicado entre los límites aguas arriba y abajo del pedregal (Figura 2). Para evitar perdida de rumbo, se utilizó cuerda guía con lastre, desde el inicio hasta el final del trazado descrito. Para cada trazo, mediante buceo a pulmón libre, se contabilizaron los ejemplares que se encontraron dentro de un cuadrante (marco metálico de un metro cuadrado, fabricado con varilla de hierro de media pulgada), el cual se colocaba de manera sucesiva siguiendo la línea de cada trazo y la cuerda guía. Así se estableció la densidad y la distribución de los peces en cada ocasión. A efectos de cotejar si había diferencia en la densidad al inicio de la temporada de pesca y al final de la misma, se compararon los registros por metro cuadrado en cada temporada mediante ANOVA con $\alpha=0.05$. El patrón de distribución de la especie en el pedregal se determinó utilizando la razón varianza $\left(s^{2}\right)$ : media $(\bar{x})$, siguiendo el criterio tradicional que considera que si la relación es igual a 1 la distribución es aleatoria, si es igual a 0 es uniforme y si es mayor a 1 es agregada o de contagio (Balán-Dzul y Jesús-Navarrete, 2011).

En cada muestreo realizado, se tomaron datos de temperatura, $\mathrm{pH}$ y oxígeno disuelto con una sonda multiparamétrica digital (YSI 100, USA) y transparencia con disco Secchi, a nivel superficial y profundo, cada 2 metros desde la orilla hasta que el pedregal finalizaba en el centro del río; estableciéndose las diferencias entre los registros superficiales y profundos mediante ANOVA.

Definición de parámetros reproductivos de la especie. Con frecuencia mensual se realizaron salidas al pedregal con el apoyo de los pescadores, en ellas se colectaron ejemplares de la especie, estimando el tamaño mínimo de muestra en 10 individuos.
Cada ejemplar capturado fue anestesiado con benzocaina al $10 \%$, hasta la pérdida del reflejo caudal, luego medida la Longitud Estándar (LE), con un calibrador pie de rey con 0,001 $\mathrm{mm}$ de aproximación, y pesado en una balanza digital con 0.1 g de aproximación. Una vez medidos los peces fueron sacrificados y enseguida disectados para determinar el sexo y estado de madurez gonadal que siguió la escala de 1 a 4, donde, 1 correspondía a ejemplares inmaduros, 2 en maduración, 3 maduros y 4 desovados (Vazzoler, 1996; Bustamante et al., 2010).

Para la determinación de la época de madurez, se elaboraron histogramas de frecuencia de ejemplares de los cuatro estados, estimando como época de reproducción aquella en la que se observó mayor proporción de ejemplares maduros y desovados con relación al número total de ejemplares muestreados (Vazzoler, 1996).

La proporción sexual de la muestra obtenida se calculo mediante la distribución de frecuencias relativas mensuales de machos y hembras; utilizando el Test exacto de Fisher para probar la hipótesis de relación 1: 1.

La talla media de los ejemplares maduros $\left(\mathrm{L}_{50 \%}\right)$ se estimó de acuerdo con la metodología utilizada por Borda y Cruz (2004), en la cual las tallas de los ejemplares maduros se agruparon en nueve intervalos de clase, a los cuales se les estableció la frecuencia relativa y la frecuencia relativa acumulada $\left(f_{R A}\right)$.

Para estimar los parámetros a y b, se procedió a linealizar la ecuación transformando la frecuencia relativa acumulada mediante la ecuación:

$$
T f_{R A}=\operatorname{LN}\left(1 / f_{R A}-1\right)
$$

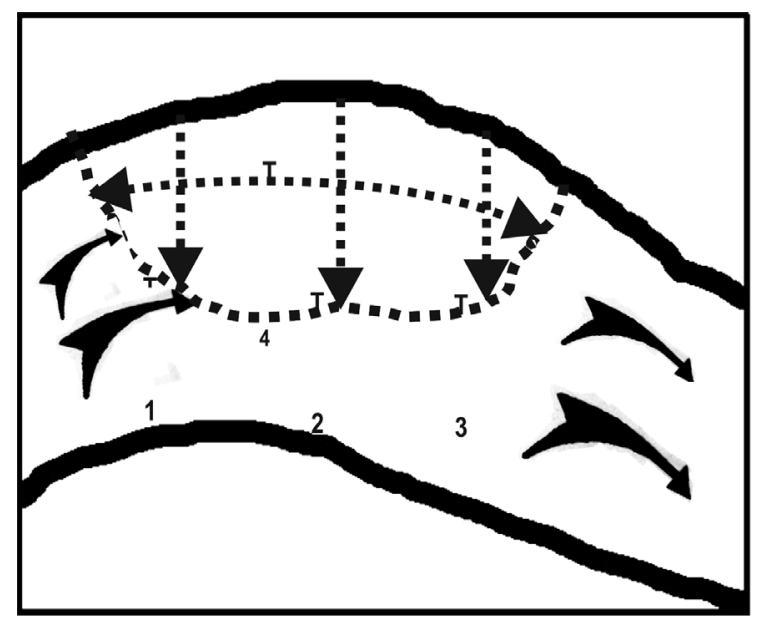

Figura 2. Esquema de los transeptos guías para cuantificación de cuchas rojas Hemiancistrus guahiborum en el pedregal El Alizal del río Bita, Puerto Carreño - Colombia. Flechas indican sentido de la corriente.. 
Donde $\mathrm{Tf}_{\mathrm{RA}}$ es la transformación de la frecuencia relativa acumulada; LN es logaritmo natural.

Posteriormente se calculó la regresión lineal entre la marca de clase y la transformación de la frecuencia relativa acumulada para determinar los parámetros a y b que se utilizaron para trazar la curva logística:

$$
P=\left(1+\mathrm{e}^{\left(\mathrm{a}-\left(\mathrm{b}^{*} \mathrm{LE}\right)\right)}\right)^{-1}
$$

Donde:

$\mathrm{P}$ es la proporción de individuos a una determinada talla; LE es la marca de clase; a es el intercepto de la regresión y $\underline{b}$ es la pendiente de la regresión.

Así se trazó la curva, donde la marca de clase se presenta en el eje de las abscisas y $\mathrm{P}$ en el eje de las ordenadas. La talla al $50 \%$ se considera la $\mathrm{L}_{50 \%}$.

A las hembras maduras halladas en cada muestreo, además, se les extrajeron los ovarios los que fueron pesados de manera precisa en balanza analítica (Memettler Toledo AB 204S, 0.0001 g de sensibilidad) y luego sumergidos en fluido de Gilson. La fecundidad absoluta $(F)$, fue determinada mediante conteo bajo estereoscopio de los ovocitos observados de seis hembras. La Fecundidad Relativa a Peso $\left(F_{R P}\right)$ fue calculada conforme la fórmula $F_{R P}=F / P(g)$ expresada como ovocitos por g de peso corporal.

Muestras equivalentes al $10 \%$ de cinco de los ovarios fueron fijadas en formol al $10 \%$ por ocho horas, conservadas en etanol al $70 \%$ y procesadas histológicamente con coloración hematoxilina - eosina.

Los diámetros de los ovocitos (Do), de cinco de los ovarios en fresco fueron medidos con calibrador de 0,01 mm de precisión.

También se estimo el factor de condición total $(K)$, el factor de condiciónsomático $\left(K^{\prime}\right)$ y el índice gonadosomático (Ig) de las hembras, para todos los meses en los que se obtuvieron ejemplares, de acuerdo con la metodología propuesta por Vazzoler (1996). Los valores promedios mensuales de estos parámetros fueron graficados y analizados mediante ANOVA, para observar su dinámica en el ciclo estudiado.

Para todos los análisis estadísticos que se realizaron se utilizó el paquete estadístico PAST 2.12 (Hammer et al., 2001) con significancia $\alpha=0,05$.

\section{Resultados}

El Alizal es un pedregal típico de la región localizado a $21.3 \mathrm{~km}$ aguas arriba de la desembocadura del río Bita en el río Orinoco $\left(6^{\circ} 12^{\prime}\right.$ 0,8 $\left.8^{\prime \prime} \mathrm{N} ; 67^{\circ} 38^{\prime} 20,5^{\prime \prime} \mathrm{O}\right)$. Posee una estructura heterogénea constituida por rocas porosas intercaladas con rocas solidas, todo lo cual le posibilita gran oferta de nichos a la fauna que lo habita. El agua del río que lo baña es cálida con temperatura de $30.9 \pm 0.3^{\circ} \mathrm{C}$, máxima $31.1^{\circ} \mathrm{C}$ y mínima de 30.1 ${ }^{\circ} \mathrm{C}$, excelentemente oxigenadas de $8.2 \pm 0.1 \mathrm{mg} \mathrm{O}_{2} / \mathrm{L}$, ligeramente acidas $\mathrm{pH} 6.6 \pm 0,1$ y con transparencia superior a $3 \mathrm{~m}$, siendo que no existen diferencias estadísticas significativas entre el valor de las mediciones de las aguas superficiales y profundas $(\mathrm{P}>0.05)$.

La cucha roja vive en El Alizal a densidad de 0.06 individuos $/ \mathrm{m}^{2}\left(\mathrm{n}=218\right.$, varianza $\left.\left(\mathrm{s}^{2}\right)=0.52\right)$, sin que se encontrará diferencia estadística $(\mathrm{P}>0.05)$ entre la densidad al inicio y al final de la temporada de pesca. El patrón de distribución es agregado o de contagio $\mathrm{s}^{2} /=8.86$, que significa que se distribuye en sitios preferenciales dentro del pedregal.

La unidad económica de pesca (UEP), está constituida por un pescador y su careta de buceo, siendo la captura de los peces manual por inmersión. Los pescadores no requieren embarcación para llegar al sitio de captura ya que acceden a él por vía terrestre.Para las faenas de pesca salen grupos de dos o tres pescadores pero la captura propiamente dicha es independiente y cada uno mediante buceo a pulmón ubica y captura los peces en las grietas. Cuando no puede tomar los peces de los orificios de las rocas entonces las remueve y las fracciona golpeándolas entre sí para conseguir capturarlas. La actividad pesquera está condicionada por el nivel y transparencia del río, por lo que la temporada de mayor captura se realiza en la estación de aguas bajas siendo que para el estudio que se reporta esta se inicio el 15 de enero y finalizo a mediados de abril de 2009.

La faena de una UEP dura $52 \pm 20$ minutos, con un mínimo de 25 inmersiones y tiempo de inmersión de $48 \pm 24$ segundos $(n=139)$. Se registro una captura de $28 \pm 18$ peces por UEP.

Durante los 12 meses de estudio se muestrearon 150 ejemplares con talla de 4 a $12 \mathrm{~cm}$ de LE (tabla1). La proporción sexual se estableció de 1 hembra: 1,5 machos, con diferencia significativa $(\mathrm{P}<0.05)$, rechazando la hipótesis de una proporción 1:1. La ausencia de muestras entre junio y septiembre se debió a la alta turbidez y profundidad del afluente que impidió las faenas de pesca.

En los meses muestreados se observaron ejemplares en todos los estados de madurez gonadal siendo que se hallaron 32 individuos maduros, variando la propor- 
Tabla 1. Ejemplares de Hemiancistrus guahiborum muestreados por marca de clase de longitud estándar (LE) por meses durante el período de muestreo. $n=150$

\begin{tabular}{|c|r|r|r|r|r|r|r|r}
\hline $\begin{array}{c}\text { Marca de clase de LE } \\
(\mathbf{c m})\end{array}$ & Ene & Feb & Mar & Abr & May & Oct & Nov & Dic \\
\hline 4 & 0 & 0 & 1 & 0 & 0 & 0 & 4 & 0 \\
5 & 5 & 10 & 0 & 2 & 0 & 0 & 1 & 2 \\
6 & 5 & 3 & 4 & 3 & 2 & 4 & 3 & 4 \\
7 & 12 & 6 & 11 & 4 & 4 & 6 & 1 & 2 \\
8 & 1 & 0 & 9 & 0 & 9 & 0 & 2 & 1 \\
9 & 1 & 0 & 9 & 1 & 3 & 1 & 0 & 0 \\
10 & 0 & 1 & 3 & 3 & 2 & 0 & 1 & 1 \\
11 & 0 & 0 & 1 & 0 & 1 & 0 & 0 & 0 \\
12 & 0 & 0 & 0 & 1 & 0 & 0 & 0 & 0 \\
Total & $\mathbf{2 4}$ & $\mathbf{2 0}$ & $\mathbf{3 8}$ & $\mathbf{1 4}$ & $\mathbf{2 1}$ & $\mathbf{1 1}$ & $\mathbf{1 2}$ & $\mathbf{1 0}$ \\
\hline
\end{tabular}

ción de estos con respecto a los inmaduros de $10 \%$ en febrero a $50 \%$ en octubre (fig. 3).

En la figura 4 se muestran las estimaciones mensuales de $K$ y K', sin que se presentara diferencia significativa entre estos dos parámetros entre los meses y tampoco entre estados de madurez gonadal $(\mathrm{P}>0.05)$.

El índice gonadosomático fluctúo entre 0,3 en el mes de mayo y 8,1 en el mes de diciembre (fig. 5), encontrándose que no hubo diferencias significativas entre los valores mensuales $(\mathrm{P}>0.05)$, por la alta variabilidad en los datos mensuales.

El rango de tallas encontrado para las hembras maduras estuvo entre 4.4 y $9.1 \mathrm{~cm}$ de LE, estimándose la talla $\mathrm{L}_{50 \%}$ de madurez gonadal para ellas en $6.4 \mathrm{~cm}$ de LE. Para los machos maduros las tallas encontradas oscilaron entre 5 y $12 \mathrm{~cm} \mathrm{LE}$, con $\mathrm{L}_{50 \%}$ de madurez gonadal estimada en $7.5 \mathrm{~cm}$.

En los ovarios en fresco se observaron ovocitos grandes de varios tamaños, sin necesidad de equipos de

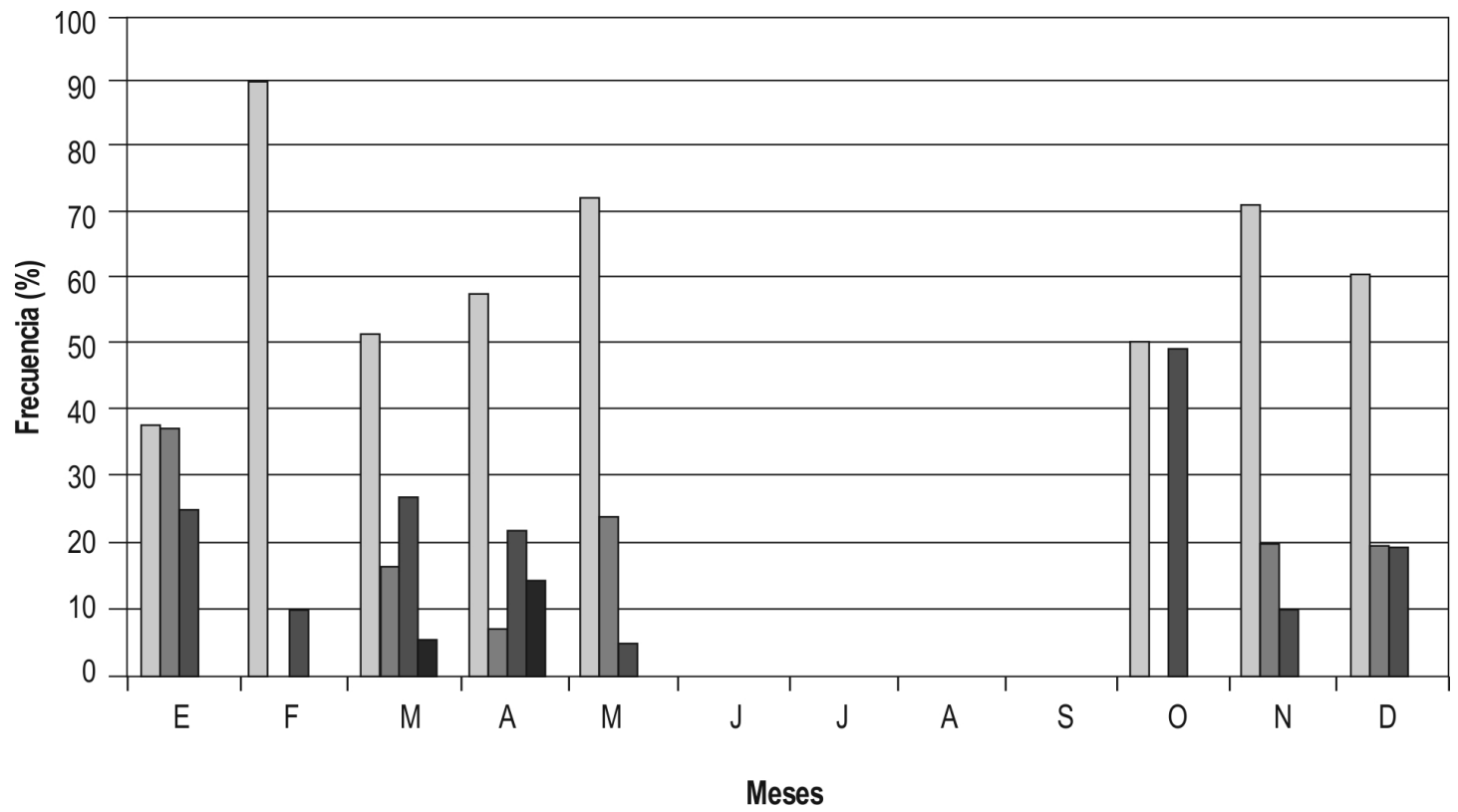

Figura 3. Frecuencia de ejemplares inmaduros, en maduración, maduros y desovados de H. guahiborum. 


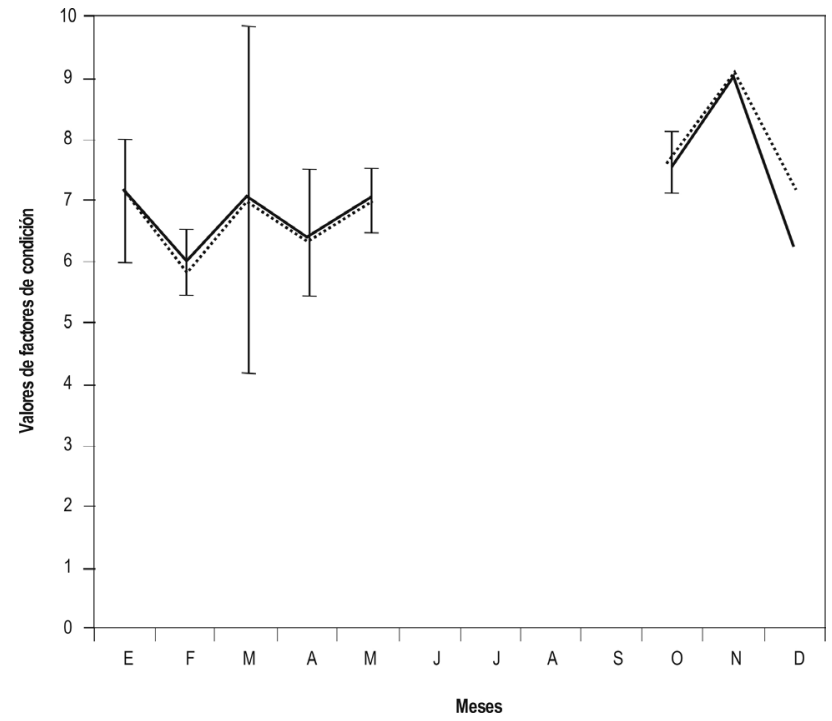

Figura 4. Variación mensual del factor de condición total $(K)$ _ y el factor de condición somático (K') - - - de hembras de $H$. guahiborum muestreadas en el ciclo hidrológico 2009, en el río Bita. En junio, julio, agosto y septiembre no se obtuvieron muestras. Valores mostrados como media \pm SD.

microscopía, durante todos los muestreos, los ovocitos maduros tuvieron diámetro de $2.3 \pm 0.4 \mathrm{~mm}(\mathrm{n}=$ 39).En los cortes histológicos se evidenciaron ovocitos maduros, en vitelogénesis exógena y endógena y ovocitos perinucleolares de distinto tamaño, lo que sugiere condición asincrónica para la especie.

La fecundidad absoluta fue $F=48 \pm 19$ ovocitos, variando entre 39 y 143 ovocitos por hembra. La $F_{R P}$ fue calculada en 5.8 ovocitos / g de hembra.

\section{Discusión}

Los pedregales encontrados en el tramo estudiado se observaron en ambas orillas del río, sin que se distinguiera algún patrón en la distribución o tamaño de los mismos, esto como consecuencia de su formación a partir de eventos de erosión eólica y de lluvias que dejan al descubierto la coraza laterítica de donde se desprenden fracciones que caen al cauce y ya en el río la corriente contribuye a modelar la aglomeración

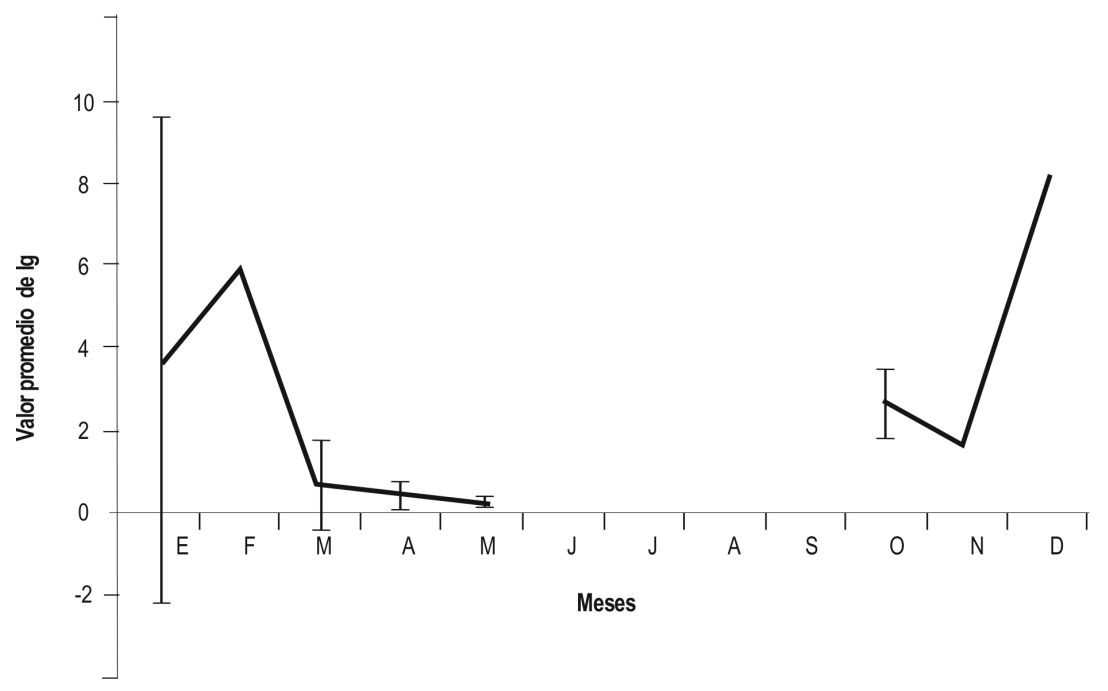

Figura 5. Variación mensual del Índice gonadosomático ( $\mathrm{Ig}$ ) de hembras de H. guahiborum muestreadas en el ciclo hidrológico de 2009 en el pedregal El Alizal en el río Bita. Sin muestras en los meses de junio, julio, agosto y septiembre. Valores mostrados como media \pm SD. 
rocosa con zonas de mayor concentración de piedras y espacios vacios en los que se observa la arena del fondo del río.

La transparencia y $\mathrm{pH}$ del agua en la zona de estudio, permiten considerar al río Bita como un río de aguas ácidas y claras propias de los afluentes que nacen y recorren la altillanura de la Orinoquía en Colombia, con aguas transparentes en verano y turbias en invierno (Rosales et al., 2010). El nivel de oxígeno encontrado es superior al reportado por Ramírez-Gil y Ajiaco-Martínez (2001), para el Orinoco y cuerpos de aguas asociados al mismo, con un valor máximo de $6.9 \mathrm{mg} / \mathrm{L}$. Con relación a la temperatura, se observa que es similar a la registrada por Maldonado (1999), citado en Galvis et al. (2007), en el mismo pedregal en enero de 1998, de $31{ }^{\circ} \mathrm{C}$. La similitud de los registros de los parámetros medidos en la superficie y el fondo se debe a la mezcla de la columna de agua producto de la velocidad, turbulencia y poca profundidad de sitio.

La distribución de tipo agregado que presenta la especie en el pedregal, es similar a la descrita por otros autores para otras especies de peces en otros ecosistemas. En general esta distribución se puede caracterizar porque los individuos se encuentran en grupos o parches lo que indica interrelaciones entre los individuos o entre los individuos y el medio, que pueden estar ligadas con interacciones sociales, modo reproductivo, recursos alimenticios, comportamientos defensivos, áreas de mayor corriente y parches de calidad, entre otros (Márquez, 2000). Nieto et al. (2006), sugieren que el patrón de agregación puede estar relacionado además con factores ambientales como temperatura, altitud, concentración de oxígeno y velocidad de la corriente, entre otros, en tanto que Palacio-Nuñez et al. (2010), al analizar la ictiofauna del Lago Media Luna en México, reportan que las relaciones con otras especies, la presencia de vegetación, la competencia y el tamaño, también influyen en la distribución agregativa de las especies.

Como a lo largo de los transeptos analizados los valores de calidad de agua fueron homogéneos, se puede considerar que estos no son del conjunto de factores que influyen en el comportamiento agregativo en este caso, lo que lleva a pensar que la distribución que se reporta podría estar establecida por el aprovechamiento de los espacios en las rocas que son utilizados como refugio para protegerse de los predadores y también para establecer sus nidos, pudiéndose asumir que el comportamiento gregario con mayor concentración de ejemplares en las zonas donde se presente mayor disponibilidad de rocas porosas y grietas sea la razón principal de la distribución descrita. Si bien en este trabajo no se caracterizó el tipo de sustrato de cada cuadrante, se pudo observar que en los cuadrantes constituidos por suelos de arena, sin rocas, no se capturaban ejemplares de la especie, lo anterior, para corroborar lo antes señalado. Con todo, es necesario hacer estudios más precisos que permitan correlacionar la distribución tanto con los factores antes expuestos como con otros como, la luminosidad, la talla de los peces, la presencia de otras especies y la oferta de alimento.

Por el tipo de distribución de la especie, no es posible, con la metodología empleada, estimar su abundancia absoluta en el pedregal. Utilizar la CPUE, como indicador de abundancia relativa, no parece apropiado pues en este caso no es posible establecer con antelación la relación entre este índice y el volumen de la población (FAO, 2001), dado que la captura de la especie está afectada por el comportamiento agregativo de la misma, similar a lo señalado por Paloaheimo y Dicky (1964) en Petitgas (1998) en otras pesquerías, al igual que por variables técnicas y ambientales, como la pericia del pescador, la intensidad de la luz que cambia en el transcurso del día de acuerdo con la posición del sol en el horizonte, con la nubosidad y con la transparencia que varía entre días, factores que generan alta dispersión en las cantidades capturadas, como se observó en el estudio y que hacen difícil estandarizar las condiciones de las faenas para poder hacer comparaciones entre las mismas.

En el período estudiado, al no encontrar diferencia significativa entre los resultados de densidad y distribución obtenidos en el muestreo al inicio de la temporada de pesca y el realizado al finalizar la misma, se podría pensar que las faenas de pesca que se hicieron en ese lapso no afectaron ni la densidad ni el tipo de distribución de los peces en el pedregal. Sin embargo, para poder confirmar esta hipótesis será necesario realizar el mismo tipo de evaluación en un pedregal que no haya sido objeto de explotación y observar si el comportamiento es similar y en caso de ser diferente, las posibles causas de la divergencia, dentro de las cuales podría ser la presión de pesca entre otras.

La pesca mediante buceo a pulmón ha sido reportada en el área de influencia de Puerto Carreño para otras especies de loricáridos como cucha piña (Panaque sp.), cucha guacamaya (Peckoltia sabaji) y cucha cebra (Peckoltia vittata) por Ramírez-Gil et al. (2001), de acuerdo con estos autores el 5\% de la captura total de peces ornamentales en la región es obtenida de esa manera. Si bien este método de captura presenta limitaciones a los pescadores porque se requiere de condiciones ambientales especiales para las faenas, 
durante el estudio se obtuvieron muestras en los períodos de aguas descendentes, bajas y ascendentes, tres de los cuatro períodos hidrológicos en los que siguiendo el patrón de inundación se puede dividir el ciclo anual en estas latitudes.

En los muestreos de ejemplares para los estudios biológicos, la proporción 1:1.5 que se reporta, debe tomarse con reserva y no asumirse como la proporción de la población, pues son necesarios estudios más profundos de dinámica poblacional y de presión de pesca, porque factores como el tamaño o la ubicación en el pedregal pueden condicionar la mayor frecuencia de pesca sobre machos que sobre hembras y así distorsionar las proporciones sexuales.

El hecho de encontrar en todos los meses ejemplares maduros, como se observa en el histograma de frecuencias, unido al comportamiento del factor de condición ( $\mathrm{K}$ y $\mathrm{K}^{\prime}$ ) y del índice gonadosomático, sin variaciones significativas entre meses, junto con la presencia de diferentes fases de desarrollo de los ovocitos, como se preciso, sugieren la condición asincrónica de la especie, con desoves parciales a lo largo del año. Trabajos en el futuro con un mayor número de ejemplares y durante todos los meses del año deberán hacerse para ser concluyente este aspecto y asegurar que los cambios en los niveles del río, contrario a lo registrado con otras especies de loricáridos en la Orinoquia colombiana, como Dekeyseria pulcher, que se reproduce solo en la temporada de aguas ascendentes (Ruíz-Vanegas et al., 2001), no son determinantes para la especie.

De otra parte la presencia de ejemplares maduros durante todo el estudio podría explicar la hipótesis propuesta por Agostinho et al. (1991), para Hypostomus commersoni, quienes sostienen que la ocurrencia de ejemplares maduros durante largo tiempo se debe a una estrategia de maduración por grupos para disminuir la competencia intraespecífica e interespecífica por los sitios de anidación y desove, que sería como el caso que se discute, dado que la especie usa cavidades en la roca para el cuidado de sus huevos y crías.

Los machos maduros observados tenían mayor longitud que las hembras maduras, lo que se refleja en un mayor $L_{50}$, para ellos, sin que se pueda establecer si esta diferencia en tamaño se deba a un dimorfismo sexual secundario o que simplemente los machos son más longevos que las hembras, lo que les permite alcanzar más talla. Este mayor tamaño de los machos podría relacionarse también con lo expuesto por Dei et al. (1997), quienes consideran que en los machos cuidadores el tamaño es una ventaja al momento de proteger los huevos y larvas contra predadores.
La estrategia de cuidado de las crías, unida a huevos grandes, como se reporta, son de las más conocidas explicaciones para la baja fecundidad hallada, esto es particularmente cierto para especies de loricáridos como lo describe Suzuki et al. (2000), para L. platymetopon con diámetros ovocitarios de $2.3 \mathrm{~mm}$ y fecundidad relativa de 9.5 ovocitos/g y Loricariichthys sp. con diámetros ovocitarios de $2.7 \mathrm{~mm}$ y fecundidad relativa de 5.7 ovocitos/g, y en las que ambas exhiben cuidado parental, en contraste, especies con ovocitos pequeños y sin cuidado parental, tienen mayor fecundidad, como el caso de otro loricárido, Rinolepsis aspera con diámetro ovocitario de $1.2 \mathrm{~mm}$ y fecundidad relativa de 39.9 ovocitos/g (Suzuki et al., 2000). El modo reproductivo con posturas en nidos y cuidado parental también promueve el comportamiento de distribución agregado.

Esta especie además de ser un componente del ecosistema del río Bita, presta unos beneficios sociales y económicos a la comunidad de Puerto Carreño, ya que su aprovechamiento comercial genera empleo e ingresos a los habitantes del lugar; en este sentido, es necesario el ordenamiento de su explotación, para mantener un adecuado nivel de producción, que permita obtener a los pescadores y comerciantes un ingreso razonable, pero que no atente contra la estabilidad tanto de este recurso pesquero como del ambiente en el que se encuentra.

Las estrategias clásicas de ordenación pesquera están relacionadas con la limitación al acceso a los recursos, la regulación de artes de pesca y el establecimiento de lugares y zonas de veda (Kapetski, 1982), a estas los nuevos conceptos incorporan las estrategias empleadas por la comunidad de manera espontánea (Scudder y Conelly, 1986), en el entendido que sin la participación directa de los usuarios del recurso la aplicación de cualquier medida no daría los resultados esperados.

Si bien, los aspectos socioeconómicos y ecológicos de esta pesquería, no fueron objeto de estudio en este trabajo, si se pudo establecer que en la zona ni los pescadores ni los comerciantes tienen alguna consideración especial sobre la explotación, la idea es capturar todo lo que se requiera, sin importar lo que pueda ocurrir con la especie o con el ecosistema, por lo que la primera propuesta para avanzar en el manejo adecuado de la especie es la implementación de una estrategia de capacitación y concientización de la comunidad acerca del aprovechamiento del recurso que lleve a la construcción de acuerdos entre los usuarios y entre los usuarios y las autoridades pesqueras y ambientales.

Welcomme (1980), citado en Scudder y Conelly (1986), considera que el control a las temporadas de 
pesca es una de las técnicas de ordenación más seguras para las autoridades y se ha empleado de manera regular en pesca de consumo; las vedas también han sido propuestas como estrategia de manejo y conservación de los peces ornamentales de la región de Sudamérica (INCODER et al., 2006). Esta especie ya tiene una veda natural, de aproximadamente cuatro meses, debido a que para su captura se requieren condiciones especiales de transparencia del agua que solo se dan en el verano como quedo demostrado, por lo que la presión de pesca se ejerce en un período muy corto y dado que los resultados indican que la especie se reproduce durante todo el año, es de esperar que en esa temporada los procesos de reproducción se adelanten de manera exitosa, permitiendo la renovación de las poblaciones. Adicionalmente en la zona ya está reglamentada una veda al acopio, transporte y comercialización de peces ornamentales durante los meses de mayo y junio, que significa una suspensión total de la actividad pesquera, en la que está incluida $H$. guahiborum, medida que se debe mantener, para aumentar el espacio de tiempo sin intervención sobre las poblaciones.

La regulación al tamaño de comercialización de los peces es otra forma de ordenamiento que se ha empleado en pesquerías de especies de consumo (Kapetsky, 1982); teniendo el reporte de la $L_{50}$ de madurez gonadal, es necesario que se evalué el establecimiento de una talla mínima de comercialización en $6 \mathrm{~cm}$ de LE, para evitar la sobrepesca al crecimiento; se sugiere esta talla que corresponde a la de las hembras, por ser ellas las más vulnerables en el período de reproducción dado el gasto energético que deben hacer para la formación de los huevos. Como otra medida para asegurar la mayor cantidad de reproducciones exitosas, se debería prohibir la captura y comercialización de hembras maduras.

De igual manera se pueden emprender acciones no regulatorias dentro de las cuales se proponen:

- El establecimiento de nuevas zonas de cría, ampliando el tamaño de los pedregales actuales en el río Bita mediante el enriquecimiento con piedras en las zonas más profundas y de fuerte corriente, aumentando así los refugios para la especie. Estos nuevos ambientes deberán ser respetados por los pescadores, a fin de garantizar la reproducción de tal forma que a partir de ellos se suplan los peces que van a ser extraídos en los demás pedregales.

- Rotación de pesca en los pedregales de común acuerdo con los pescadores, utilizando unos en una temporada de pesca y otros en la siguiente, de manera que se dé tiempo a las poblaciones afectadas a recuperarse.

- Impulsar la acuicultura de la especie, incorporando en el proceso a los pescadores, para poder seguir obteniendo los beneficios de empleo y recursos que genera la especie, pero con base en una producción sostenible en ambiente confinado; esta medida es acorde con las recomendaciones hechas por los participantes en el I Taller Internacional de Peces Ornamentales, realizado en agosto de 2005 (INCODER et al., 2006).

Como las propuestas anteriores, fueron elaboradas tomando en cuenta el punto de vista biológico, enfoque de este proyecto, es necesario que tanto las autoridades como los actores contemplen en el proceso de formular un plan de manejo para $H$. guahiborum, aspectos sociales, comerciales, económicos y ambientales de la pesquería, para que desde un abordaje ecosistémico se dé una administración adecuada no solo a este recurso, sino al ecosistema en el que habita, de modo que pueda seguir proporcionando los beneficios sociales y económicos que actualmente brinda a quienes obtienen su sustento del aprovechamiento de la cucha roja.

\section{Agradecimientos}

Los autores agradecen a las entidades que apoyaron la realización de este proyecto, Fundación Omacha y Universidad Nacional, mediante el Proyecto Estudio de la biología reproductiva e implementación de técnicas de reproducción en cautiverio para nueve especies de loricáridos ornamentales de la Orinoquia colombiana Contrato: 060-2007U6057-82-07

\section{Referencias}

Agostinho AA, Segatti N, Agostinho C. Ciclo reprodutivo e primeira maturacao de femeas de Hypostomus commersonii (Valenciennes, 1840) (Siluriformes, Loricariidae) no reservatorio Capivari-Cachoeira. Revista Brasileira de Biología, 1991; 51(1):31-37.

Armbruster J. Phylogenetic relationships of the suckermouth armoured catfishes (Loricariidae) whit emphasis on the Hypostominae and Ancistrinae. Zoological Journal of the Linnean Society, 2004;141:1-8.

Armbruster J. The genus Peckoltia with the description of two new species and a reanalysis of the phylogeny of the genera of the Hypostominae (Siluriformes:Loricariidae). Zootaxa, 2008; 1822:1-76.

Armbruster J, Werneke D. Peckoltia cavatica, a new loricariid catfish from Guyana and a redescription of $P$. braveri (Eigenmann 1912)(Siluriformes). Zootaxa, 2005; 822:1-14. 
Balán-Dzul V, Jesús-Navarrete A. Densidad, abundancia y estructura poblacional del caracol blanco Strombus costatus en el Caribe Mexicano. Revista. de Biología Marina y Oceanografía, 2011; 46(1):1-8.

Borda CA, Cruz R. Reproducción y reclutamiento del molusco Anadara tuberculosa (Sowerby, 1883) en el pacífico Colombiano. Revista de Investigaciones Marinas, 2004; 25(3):185-195.

Bustamante C, Salinas JC, Díaz- Granados MC. (Editores). 2010. Protocolo para la toma de información pesquera, biológica y socioeconómica del sector pesquero en Colombia. Bogotá, D.C., p. 40 p.

Caddy JF, Mahon R. 1996. Puntos de Referencia para la Ordenación Pesquera. FAO Documentos Técnicos de Pesca N o347. Roma, p.109.

Cardoso A, Lucinda F. Three new species of Hemiancistrus (Teleostei: Siluriformes: Loricariidae) from the rio tocantins basin with comments on the genus. Ichthyological Exploration of Freshwaters, 2003;14(1):73-84.

Cardoso A, Silva JFP. Two new species of the Hemiancistrus Bleeker (Teleostei:Siluriformes:Loricariidae) from the upper rio Uruguay basin. Neotropical Ichthyology, 2004;2:1-8.

Castro JD. 2005. Comercialización de peces ornamentales en Colombia. En: Memorias taller internacional "Aspectos socioeconómicos y de manejo sostenible del comercio internacional de peces ornamentales de agua dulce en el norte de Sudamérica: retos y perspectivas" Agosto 24, 25 y 26 de 2005. Bogotá, Colombia, p. 72.

Dei C, Agostinho AA, Suzuki HI. Population estructure and reproductive biology of Loricariichthys platymetopon (Siluriformes, Pisces) in the upper river Paraná. Brazilian archives of biology and technology, 1997; 40 (4):793-807.

FAO - Organización de la Naciones Unidas para la Alimentación y la Agricultura. 2001. Directrices para la recuperación sistemática de datos relativos a la pesca de captura. FAO Documentos Técnicos de Pesca, №382, Roma, p.132.

FAO - Organización de la Naciones Unidas para la Alimentación y la Agricultura. 2003. La ordenación pesquera. 2. El enfoque de ecosistema en la pesca. FAO orientaciones técnicas para la pesca responsable, № 4 (Suplemento 2), Roma, p.136.

Galvis G, Mojica Jl, Provenzano F, Lasso CA, Taphorn DC, Royero R, Castellanos C, Gutiérrez A, Gutiérrez M, López Y, Mesa L, Sánchez P, Cipamocha CA. 2007. Los peces de la Orinoquía colombiana con énfasis en especies de interés ornamental. Instituto Colombiano de Desarrollo Rural - Universidad Nacional de Colombia. Bogotá, D.C.,p 425.

Hammer $\varnothing$, Harper DAT, Ryan PD. PAST: Paleontological statistics software package for education and data analysis. Palaeontologia Electronica 2001; 4(1): 9pp. http://palaeo-electronica. org/2001_1/past/issue1_01.htm

INCODER - Instituto Nacional de Desarrollo Rural, Traffic-América del Sur y WWF-Colombia. 2006. Memorias taller internacional "Aspectos socioeconómicos y de manejo sostenible del comercio internacional de peces ornamentales de agua dulce en el norte de Sudamérica: retos y perspectivas" Agosto 24, 25 y 26 de 2005. Bogotá, Colombia, p. 72.
Kapetsky JM. 1982. Consideraciones para la ordenación de las pesquerías de lagunas y esteros costeros. FAO Documentos Técnicos de Pesca, №128, Roma, p. 49.

King M. 1995. Fisheries biology, assessment and management. Fishing News Book. Oxford, England, p. 341.

Maldonado-Ocampo J, Lugo M, Bogotá-Gregory JD, Lasso CA, Vásquez L Usma JS, Taphorn D, Provenzano F. Peces del río Tomo, cuenca del río Orinoco, Colombia. Biota colombiana, 2006; 7 (1):113-228.

Márquez E. 2000.Curso de Biología de Poblaciones y Evolución. Tema 2: Disposición Espacial. 12 de septiembre de 2011. URL:http.//prof.usb.ve/ejmarque/cursos/ea2181/core/ desp01.

MADR - Ministerio de Agricultura y Desarrollo Rural y CCI -Corporación Colombia Internacional, 2008. Pesca y acuicultura en Colombia 2007. Informe Técnico Regional Cuencas del Orinoco y Amazonas. Ministerio de Agricultura y Desarrollo Rural Corporación Colombia Internacional. Bogotá, D.C., p. 94.

Nieto K, Lizano M, Velasco JC. Distribución de los peces continentales de España asociada a las características físicas, meteorológicas e hidrológicas de las cuencas hidrográficas. Ecosistemas, 2006;15(1):69-76

Palacio-Núñez J, Verdú JR, Numa C, Jiménez-García D, Olmos G, Galante E. Freshwater fish's spatial patterns in isolated water springs in North-eastern Mexico. Revista de Biología Tropical, 2010; 58 (1): 413-426.

Petitgas P. Biomass-dependent dynamics of fish spatial distributions characterized by geostatistical aggregation curves. ICES Journal of Marine Science, 1998; 55: 443-453.

Ramírez-Gil H, Carrillo-Villar LM, Lacera-Padilla EA, Ajiaco-Martínez RE. 2001. La pesca de especies de interés ornamental en el área de influencia de Puerto Carreño. En: Ramírez-Gil, H y Ajiaco - Martínez RE (Editores). La pesca en la baja Orinoquia colombiana: una visión integral. INPA. Bogotá, D.C.,p. 123-138.

Ramírez-Gil H, Ajiaco-Martínez RE, 2001. La Orinoquía colombiana y su área de frontera. En: Ramírez-Gil, $\mathrm{H}$ y Ajiaco-Martínez RE (Editores). La pesca en la baja Orinoquia colombiana: una visión integral. INPA. Bogotá, D.C., p. 9-22.

Ramírez-Gil H, Ajiaco-Martínez RE. 2008. Sitios de pesca de peces ornamentales en el área de Reserva de Biosfera El Tuparro. Documento Técnico Inédito. Fundación Omacha, p. 47.

Rosales J, Suárez C, Lasso C. 2010. Descripción del medio natural en la cuenca del Orinoco. En: Lasso C, Usma JS, Trujillo F, Rial A. (Editores). Biodiversidad de la cuenca del Orinoco: bases científicas para la identificación de áreas prioritarias para la conservación y uso sostenible de la biodiversidad. Instituto de Investigación de Recursos Biológicos Alexander von Humboldt, WWF Colombia, Fundación Omacha, Fundación La Salle e Instituto de Estudios de la Orinoquia (Universidad Nacional de Colombia). Bogotá, D. C., p. 50-73.

Ruiz-Vanegas JA, Ramírez-Gil H, Ajiaco-Martínez RE. 2001. Dekeyseria pulcher Fowler, 1954. En: Ramírez-Gil, H y Ajiaco-Martínez RE (Editores). La pesca en la baja Orinoquia colombiana: una visión integral. INPA, Bogotá, D.C., p. 199-202. 
Scudder T, Conelly T. 1986. Sistemas de ordenación de las pesquerías fluviales. FAO Documentos Técnicos de Pesca, № 263, Roma, p. 50.

Seijo JC, Defeo O, Salas S. 1997. Bioeconomía pesquera - Teoría, modelación y manejo. FAO Documentos Técnicos de Pesca, № 368, Roma, p. 176.

SIPA - Sistema de información pesquero y acuícola. 2008. Ministerio de Agricultura y Desarrollo Rural - Corporación Colombia Internacional. Archivo en Excel. Bogotá, D.C.

SIPA - Sistema de información pesquero y acuícola. 2009. Ministerio de Agricultura y Desarrollo Rural - Corporación Colombia Internacional. Archivo en Excel. Bogotá, D.C.

Souza LD, Melo M, Chamon C, Armbruster J. A new species of Hemiancistrus from the rio Araguaia basin, Goias State, Bra- zil (Siluriformes: Loricariidae). Neotropical Ichthyology, 2008; 6:419-424.

Sparre P, Ursin E, Venema SC. 1989. Introduction to the tropical fish stock assessment. Part 1. Manual. FAO Fisheries Technical Paper № 306.1, Roma, p. 337.

Suzuki HI, Agostinho AA, Winemiller KO. Relationship between oocyte morphology and reproductive strategy in loricariid catfishes of the Paraná River, Brazil. Journal of Fish Biology, 2000; 57: 791-807.

Vazzoler AEA.d.M. 1996. Biologia da reprodução de peixes teleósteos: teoria e prática EDUEM, São Paulo, Brasil, p.169.

Werneke DC, Armbruster JW, Lujan NK, Taphorn DC. Hemiancistrus guahiborum, a new suckermouth armored catfish from Southern Venezuela (Siluriformes:Loricariidae). Neotropical Ichthyology, 2005; 3(4):543-548. 